\title{
Quantum Computation: from a Programmer's Perspective
}

\author{
Benoît Valiron \\ University of Pennsylvania, \\ Department of Computer and Information Science, \\ 3330 Walnut Street, Philadelphia, Pennsylvania, 19104-6389, USA \\ benoit.valiron@monoidal.net
}

Received 15 April 2012

\begin{abstract}
This paper is the second part of a series of two articles on quantum computation. If the first part was mostly concerned with the mathematical formalism, here we turn to the programmer's perspective. We analyze the various existing models of quantum computation and the problem of the stability of quantum information. We discuss the needs and challenges for the design of a scalable quantum programming language. We then present two interesting approaches and examine their strengths and weaknesses. Finally, we take a step back, and review the state of the research on the semantics of quantum computation, and how this can help in achieving some of the goals.
\end{abstract}

Keywords Quantum Computation, Models of Computation, Quantum Programming Language, Semantics.

\section{$\S 1 \quad$ Introduction}

This paper is the second part of a two-articles series. The first part ${ }^{30)}$ was concerned with a general introduction to quantum computation; in this article, we discus quantum computation specifically from a programmer's perspective.

Supported by the Intelligence Advanced Research Projects Activity (IARPA) via Department of Interior National Business Center contract number D11PC20168. The U.S. Government is authorized to reproduce and distribute reprints for Governmental purposes notwithstanding any copyright annotation thereon. Disclaimer: The views and conclusions contained herein are those of the authors and should not be interpreted as necessarily representing the official policies or endorsements, either expressed or implied, of IARPA, DoI/NBC, or the U.S. Government. 
In particular, we assume that the quantum device is already scalable and that we already know which algorithm we want to implement. The questions we want to be able to answer are how to actually write the algorithm and how to make sure that it is correct, and that it will run as expected.

The paper is organized as follows. First we discuss the distinction between the abstract models of computation in which algorithms are usually defined; then we focus on the aspect that is not addressed in these scheme, that is, the problem of error detection and correction. We then turn to the question of the programming of quantum devices, from the perspective of the discussed computational models. We first discuss the need for an actual quantum programming language: why we need one, what can we expect from it. Then we develop two attempts at building a language, and discuss their strengths, their weaknesses and what lessons can be kept for future work.

To conclude the paper, we analyze what have been done in the semantics of quantum computation: how they relate to models of computation, and what benefits these studies can bring.

\section{$\S 2$ Ideal versus realistic models of computation}

In this section, we turn to the question of setting up a robust setting for performing quantum computation. There are two sides to this coin: the first is

to define a logical layer, resilient to decoherence, and the other is the definition of a paradigm for quantum computation, with ideal quantum bits.

\subsection{Models of computations}

What are the computational paradigms that allow us to actually design and reason about algorithms? In this section, we briefly describe three of them. Although there exist (many) other models of computation (quantum Turing machine, quantum cellular automata, adiabatic quantum computation...), we only focus on the models that we feel are the most susceptible to interface between a hardware and a potential programming language.

Quantum circuits. As we saw in Section 6 (Part ${ }^{30)}$ ), many quantum algorithms consist of big blocks of preparation phase, followed by the application of a unitary map (described in term of elementary operations), terminated by the measurement of the system. The whole process is then regarded as a fancy 
probabilistic process from which one can make some sense with classical preand post-processing.

If we draw a parallel with classical boolean circuits, it makes sense to consider quantum circuit as a model of quantum computation. A quantum algorithm is then seen as a family of regular quantum circuits, one for each input size. The circuits have all the same structure:

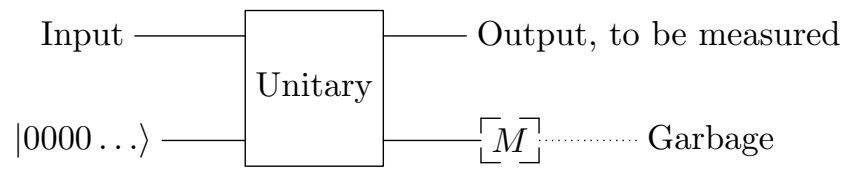

This model is the "canonical" model of quantum computation, and the expressive power of new models is usually determined by comparison with this one.

It has the advantage of being extremely close to the standard description of quantum algorithms, and it is also well-suited for some physical implementations, such as quantum $\mathrm{NMR}^{30)}$ : we first set up an array of quantum bits, then we send a series of pulses to change the state of the system, then we measure and destroy the system.

The QRAM model. The model of computation behind quantum circuit can be generalized to the model of "quantum random access machine" ${ }^{21)}$. The model can be best described as "quantum data, classical control" ${ }^{26)}$ : the computation essentially takes place in a classical computer to which a quantum device is connected. The device holds the quantum memory and can allocate new quantum bits and measure or apply unitary maps on existing quantum bits at wish. The quantum device is a black-box whose entries are instructions and whose only outputs are the results of measurements and return codes of instructions.

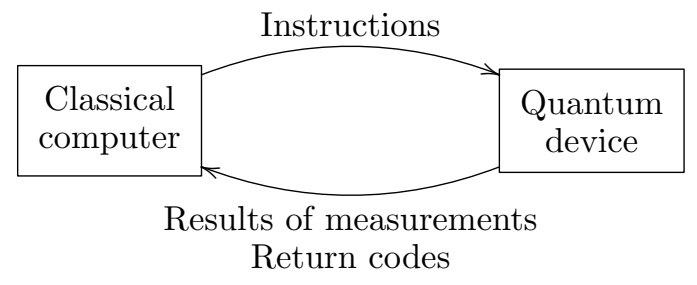

It is the model of computation implicitly behind most of the quantum algorithms we discussed so far, and behind most error correcting codes. It is a versatile model in which one can express most of the other models of quantum computations. 
Measurement-based model. Quantum circuits let us think that unitaries are the ultimate computational unit of quantum computation. It turns out that it is not the case, and one can develop a theory of computation using "mostly" measurements. The model is called measurement-based quantum computation ${ }^{24}$ (MBQC). In the following, we use the definitions found in (Danos et al., 2009) $)^{7)}$ In this model, quantum bits are usually represented arranged on a lattice, and separated in three groups: the input qubits, the outputs qubits and the auxiliary qubits. There are three main operations at hand:

- initialization of a quantum bit to the value $\frac{1}{\sqrt{2}}(|0\rangle+|1\rangle)$;

- entanglement of 2 (adjacent) quantum bits using $Z_{C}$;

- measurement against the basis $\frac{1}{\sqrt{2}}\left(|0\rangle+e^{i \alpha}|1\rangle\right), \frac{1}{\sqrt{2}}\left(|0\rangle-e^{i \alpha}|1\rangle\right)$;

- corrections: $N$ and $Z$.

The computation run as follows. First, we initialize all the non-input qubits. Then we perform entanglement between certain pairs of qubits. Finally, a series of measures and possibly correlated corrections are applied on each of the non-output qubits. The order of operation is of course important (because of the correlated corrections), and for a particular algorithm one can draw a dependence graph specifying the order in which the measurements are supposed to be executed.

This model is interesting for several reason. First, this dependence graph is a powerful tool for optimizing a computation: it gives some non-local information on what parts of the computation are unrelated (and can potentially be run in parallel), and what parts are connected. It also give a notion of flow of information (following the dependencies) that can potentially be used for optimizing the way error-correction is implemented in the hardware.

This model is also well-connected to hardware (in particular latticebased technologies, such as trapped ions or quantum $\operatorname{dots}^{30)}$ ), and thought of as a good candidate model for computations that would be more resilient to errors than plain circuit model.

Finally, the model is equivalent to the quantum-circuit model: a quantum circuit can be re-written as a measurement-based computation and viceversa. This can be seen as both a "compilation" of a quantum circuit onto a MBQC-aware hardware, or as a tool to parallelize a quantum circuit $^{6}$ : from a programmer's perspective, this model could become a precious tool for automatic code optimization in a compiler. 


\subsection{Physical versus logical quantum bits}

Although quantum hardware is tuned to make the system as precise and stable as possible, a reasonable computation is expected to outlive the coherence time of the quantum memory ${ }^{30)}$. Moreover, the elementary operations performed on the system cannot be perfect, and errors are bound to occur. Therefore, before any practical implementation of a quantum algorithm one needs to first find a way to stabilize the quantum memory by detecting and correcting the error and a way to render the operations resilient to errors.

This problem also exists in classical computation, and for example networking protocols make heavy use of repetition codes to overcome errors. However, compared to classical computation, quantum computation has peculiarities that do not make classical codes directly applicable ${ }^{22)}$.

- No-cloning. Repetition cannot blindly be applied since a piece of quantum information cannot be duplicated in general.

- Errors are continuous. In classical computation, a bit is either flipped or not. In quantum computation, errors can be more pernicious: The state $|0\rangle$ can be modified to $\epsilon|0\rangle+(1-\epsilon)|1\rangle$, for a small $\epsilon$; the state $\frac{1}{\sqrt{2}}(|0\rangle+|1\rangle)$ can be sent to $\frac{1}{\sqrt{2}}\left(|0\rangle+e^{i \epsilon}|1\rangle\right) \ldots$ This requires to adapt classical codes, but also to develop new ideas to tackle the new setting.

- Measurement are destructive. In classical error correction, checking the value of a bit is just a matter of looking at it. In quantum computation, such an operation on a quantum bit will destroy the potentially huge entangled state in which the qubit is part of. Beside, the notion of "looking at" something has to be adapted to the new setting: In the phase shift $\frac{1}{\sqrt{2}}\left(|0\rangle+e^{i \epsilon}|1\rangle\right)$, a measure in the canonical basis will not say anything useful.

For a very readable and extensive presentation of codes for detection and correction of errors, see Chapter 10 of (Kaye et al, 2007) ${ }^{20)}$.

Three-qubit bit-flip code. To illustrate the issues, we give a concrete example of error-detection and correcting code adapted from the classical world. Suppose that the errors from the environment are random application of notgates $N$ on our system: this is the bit-flip error-model.

A simple code to correct this error uses 3 qubits to encode one qubit. As for the classical case, the correction uses the majority vote to correct the error: the logical qubit $|\mathbf{0}\rangle$ is encoded with the three qubits $|000\rangle$, and $|\mathbf{1}\rangle$ with the 


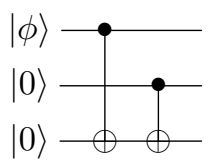

(a) Creation

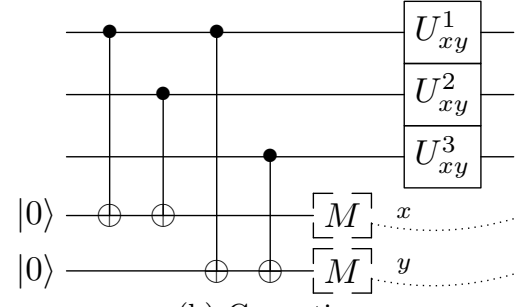

(b) Correction

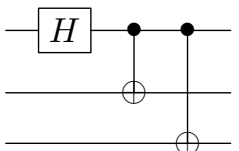

(c) Unitary

Fig. 1 Three-qubit bit-flip code

three qubits $|111\rangle$. If after a while, the logical qubit is $|001\rangle$, we can reasonably consider that we are instead looking at $|000\rangle$, that is, $|\mathbf{0}\rangle$.

Consider a quantum bit in state $|\phi\rangle$. As we just discussed, we cannot duplicate a general quantum state. Instead, we can use $N_{C}$ as in Figure 1(a). If $|\phi\rangle=\alpha|0\rangle+\beta|1\rangle$, the resulting state of the circuit is $\alpha|000\rangle+\beta|111\rangle$. We successfully encoded $|\phi\rangle$ as a logical qubit.

The next problem is to detect and recover from errors: If the encoded $|\phi\rangle$ suffers from a bit-flip and becomes $\alpha|001\rangle+\beta|110\rangle$, we need to first detect the problem, and then to correct it. But detecting it would require a measure, and a measure would destroy the state, right? The trick is to perform the detection in a unitary way, using two auxiliary qubits to store the result. From the structure of the error, we can then simply measure these qubits and applying a correcting scheme of Figure 1(b), where the gates $U_{x y}^{i}$ are adjustments to perform depending on the output of the 2 measurements: either identity or not-gate.

If we can encode "stabilized" logical quantum bits as 3 physical qubits, we also have to modify the elementary gates accordingly. For example, the Hadamard gate sending $|\mathbf{0}\rangle$ to $\frac{1}{\sqrt{2}}(|\mathbf{0}\rangle+|\mathbf{1}\rangle)$ and $|\mathbf{1}\rangle$ to $\frac{1}{\sqrt{2}}(|\mathbf{0}\rangle-|\mathbf{1}\rangle)$ physically sends $|000\rangle$ to $\frac{1}{\sqrt{2}}(|000\rangle+|111\rangle)$ and $|111\rangle$ to $\frac{1}{\sqrt{2}}(|000\rangle-|111\rangle)$; it is the circuit featured in Figure 1(c).

This technique allows us to have quantum bits stable with respect to bitflip errors provided that they do not occur "too often": between each application of the detection-recover scheme, one has to be reasonably certain that only one bit-flip can occur.

Concatenated codes. There exist other codes to correct other sort of errors. For example, the three-qubit phase flip code uses three qubits for detecting and correcting the error sending $\frac{1}{\sqrt{2}}(|0\rangle+|1\rangle)$ to $\frac{1}{\sqrt{2}}(|0\rangle-|1\rangle)$. 
When one has two codes, for example the bit-flip code and the phaseflip code, it is possible to concatenate them in the sense that the (logical) input qubit of one code will be the output of the other code. In the case of these two codes, we get the Shor code, correcting an arbitrary error happening on a logical quantum bit, by using 9 physical qubits. Although it corrects only one error at a time, this could be partially solved by concatenating yet another code. However, even for this simple concatenation, the resulting logical qubit uses 9 physical qubits. And if we were concatenating more codes, the number of qubits would be even larger.

An increase in the number of quantum bits means more memory management: more qubits need to be allocated and potentially moved around. Also, the logical elementary operations are hindered by a corresponding overhead.

Given a particular hardware, there is therefore a subtle analysis to perform: sure enough there is some decoherence going on. But if it is possible at all, what is the cost of a fault-tolerant quantum computation on this hardware? Does the concatenation of one, two, three codes helps? If it does, it has to be balanced with the increased number of physical quantum bits and physical elementary gates required for their logical alter-ego.

For a real quantum computer with several hundreds of logical qubits, this analysis will probably be daunting to perform by hand only. Dedicated software analysis tools will be needed for fine tuning the design of a code for a particular hardware combination. This is one of the place where programming techniques and analysis might end up useful, if not necessary.

\section{$\S 3 \quad$ Quantum programming}

In the discussion so far, we only hinted in a few places that formal language should probably be a good thing for the field of quantum computation. This section is the place where we really pledge for quantum programming languages: why we need them (or will soon), what can they bring, and some guidelines to follow in order to get the best out of them. We then present two existing languages with some good features but yet some drawbacks, showing what we find are good directions to follow in the quest for an "ideal" programming language.

\subsection{The need for quantum programming language}

If eventually a quantum computer with enough quantum memory is 
built, it is worthwhile to wonder how to use it. Several aspects require to be taken into consideration.

Writing of algorithms. At a high-level view, the algorithms that we described in Section 6 (Part $1^{30)}$ ) are good examples of informally described algorithms: no parser will ever be able to automatically transform the textual description into an actual sequence of elementary operations.

There is therefore the need for a reasonably rich language for describing quantum computation. At a minimum, the language should have the abilities ${ }^{21)}$ :

1. to allocate quantum registers, to apply unitaries and to measure quantum registers;

2. to reason about quantum subroutines: to reverse a subroutine and to condition it over the state of a quantum register;

3. to build a quantum oracle out of the description of a classical function.

Specification and verification. In classical programming languages, debuggers are useful tools while writing programs. In quantum computation, due to impossibility to watch quantum information without modifying it, a debugger for a program is a lost cause.

In classical programming languages, one can develop test suites to analyze the behavior of a program and check whether it (statistically) behaves correctly in the expected input data-range. In the case of embedded software, if it is not possible to do it with the actual device, one can usually perform these tests off-site. In quantum computation, because of the cost of running code early quantum computers and the impossibility to efficiently simulate quantum computation on large inputs, test suites are not an option.

In typed classical programming languages, one has the choice between strong type systems, dynamic type systems, or even blends of these, bearing the fact that run-time errors can be captured and potentially resolved by the user (as in LISP, for example). In quantum computation, due to the cost of the run of a quantum computation and the difficulty to keep stable quantum memory, the programmer cannot afford run-time errors in his code, specially if they come from something as simple as the attempt to clone a quantum bit.

The conclusion is that the work have to be done upstream.

- A program needs to be specified and verified beforehand. A quantum programming language therefore needs to come up with a well-defined 
and sound semantics, in which one can specify a particular behavior for a piece of code, and eventually prove that the code behave as expected.

- Because of the cost of each run, the compiler of the programming language should catch most of the standard causes of run-time errors: the language needs a strong type system.

Resource sensitivity. At first, quantum computers will probably not have a very large quantum memory. The programming language should come up with tools to estimate the resources needed to run a particular piece of code: whether it is the number of required quantum bits or the number of elementary gates to be applied.

A particular issue in that respect is the place of the error detectioncorrection scheme: should it be managed at the code level, so that the programmer can potentially choose which code to use or apply some smart optimization, should it be left to the compiler, or should it be implemented directly in the quantum device? A programming language for quantum computation should be designed in order to be able to deal with codes for error management.

Existing languages for quantum computation. Various quantum programming languages have been proposed in the literature ${ }^{14)}$, both imperativestyle and functional-style. Many of these programming languages are theoretical apparatuses to explore particular aspects of quantum computation and not designed as scalable tools.

First, there is a trend of works developing notations for quantum Turing machines, one of the paradigms we did not discuss here: its main use is of theoretical nature.

In the realm of imperative programming languages, it is worthwhile mentioning the first real quantum programming language, $\mathrm{QCL}^{23)}$, designed by Ömer between 1998 and 2004. This fine piece of software proposes a C-like language with many nifty features for what he calls "structured quantum programming". The result is a language with a relatively natural way of writing algorithms. However, with respect to the list of requirements we developed for a successful programming language, QCL fails short in the specification/verification aspect, and in the ability to address resource sensitivity. Various other imperative languages follows QCL footsteps. In particular, a $\mathrm{C}++$ extension $^{4)}$ providing a hint at a compilation process, the command-guarded language $\mathrm{qGCL}^{25)} \ldots$ 
In the realm of quantum functional programming, the proposed languages are usually coming up with a semantics: either denotational, as the first-order $\mathrm{QPL}^{26)}$ and the quantum lambda-calculus described in Section 3.2, or operational, as van Tonder's calculus ${ }^{31)}$, or $\mathrm{QML}^{2)}$, a purely quantum language with compilation in term of quantum circuits, or the line of works proposing an embedded language in Haskell and from which Section 3.3 is derived.

In the following, we discuss two approaches that we feel partially answer the requirements we discussed in this section, and that might serve as a base for future approaches: the quantum lambda-calculus of Selinger and Valiron ${ }^{28)}$ and the Haskell-embedded quantum IO monad of Green and Altenkirch ${ }^{17)}$. Their interest lies in the fact that despite their shortcomings, they are not only rea-

sonably expressive but also they possess a well-defined and extendable semantics offering the premises of a specification.

\subsection{A toy language for the QRAM model}

We first present a minimal ML-style language for the QRAM model ${ }^{28)}$. The intuition is the following.

1. The language should be able to express any classical, boolean computation; it should be higher-order and contain pairing constructs; it should contain term constructs to express quantum bit creation, unitaries and measurements.

2. Quantum data should be a first-class citizen: one should be able to manipulate classical and quantum data with the same term constructs; variables holding quantum data should be no different from variable holding classical data; quantum data should be usable at higher-order.

3 . The language should be typed and verify usual safety properties, plus the additional requirement that it should forbid non-duplicable data to be duplicated.

4. The only side-effect should come from the probabilistic measurement. In particular, one should not need particular type constructors to hold quantum data.

The language is formally defined as a lambda-calculus. A lambda-term is an expression made of the following core grammar, written in BNF form:

$$
\begin{aligned}
\text { Term } M, N, P::= & x|M N| \lambda x . M \mid \text { if } M \text { then } N \text { else } P|0| 1|*| \\
& \text { meas } \mid \text { new }|U|\langle M, N\rangle \mid \text { let }\langle x, y\rangle=M \text { in } N,
\end{aligned}
$$


where $x$ ranges over an infinite set of term variables and $U$ a set of unitary gates; the term if $M$ then $N$ else $P$ stands for a test on $P$ with output $M$ if true, $N$ else; the constant terms 0 and 1 stand for the boolean values false and true respectively; $*$ is the result of a command; the constant terms meas, new and $U$ stand respectively for the measurement, the creation and the application of a unitary gate on a quantum bit; the terms let $\langle x, y\rangle=M$ in $N$ stands for the retrieval of the content of a pair.

We identify terms up to $\alpha$-equivalence, defined as usual ${ }^{3)}$. Similarly, the notions of free and bound variables and of substitution are standard. We use syntactic sugar for programs when the context is clear: let $x=M$ in $N$ for $(\lambda x . N) M$, and $\lambda\langle x, y\rangle . M$ for $\lambda z$. (let $\langle x, y\rangle=z$ in $N)$.

This language is purely functional: for example, a fair coin can be implemented as the term meas $(H($ new 0$))$, where $H$ is the Hadamard gate.

\section{[3.2.1] Abstract machine}

Although we want the language to perform quantum computation, no quantum bit was added into the terms of the language. For example we might want to represent the constant function outputting a quantum bit, as $\lambda x \cdot(\alpha|0\rangle+$ $\beta|1\rangle)$. The reason why we do not allow such a notation is the problem of entanglement. As discussed in the first article of this series ${ }^{30)}$, it is not always possible to write a two quantum bit system in the form $|\phi\rangle \otimes|\psi\rangle$. Therefore, if $x$ is the variable corresponding to the first quantum bit and $y$ the one corresponding to the second in an entangled state, it is not possible to write classically the term $(\lambda f . f x)(\lambda t .(g y) t)$ as there is no means of writing $x$ and $y$.

This non-local nature of quantum information forces us to introduce a level of indirection for representing the state of a quantum program. Following the scheme of the QRAM model, we describe an abstract machine consisting of a lambda-term $M$, an array of quantum bits $Q$ (the QRAM), and a map linking variables in $M$ to quantum bits in $Q$. Formally, an abstract machine is a triple $[Q, L, M]$, where

- $Q$ is a normalized vector of $\otimes_{i=1}^{n} \mathbb{C}^{2}$, for some $n \geqslant 0$, called the quantum array.

- $M$ is a lambda term,

- $L$ is an injective function from a set $|L|$ of term variables to $\{1, \ldots, n\}$, where $F V(M) \subseteq|L| . L$ is called the linking function.

We write $|Q|=n$. If $L\left(x_{i}\right)=i$, we will sometimes write $L$ as the ordered list 
$\left|x_{1} \cdots x_{n}\right\rangle$. The idea is that the variable $x_{i}$ is bound in the term $M$ to qubit number $L\left(x_{i}\right)$ of the state $Q$. We also call the pair $(Q, L)$ a quantum context. If there are no quantum bits, i.e. if $Q \in \mathbb{C}$, we write $Q=|\rangle$. Similarly, if $L$ is empty we write $L=|\rangle$.

\section{[3.2.2] Evaluation Strategy}

Although we solved the problem of entanglement, there is another issue that prevents us from blindly using substitution, namely the probabilistic nature of the measurement: since tossing a coin and duplicating the result is not the same thing as duplicating the coin and tossing each instance, we need to make a choice of reduction strategy.

A reduction strategy is a deterministic procedure for reducing a general abstract machine to a result. There are two main reduction strategies for the lambda-calculus: in the presence of $(\lambda x . M) N$, either we blindly substitute $N$ for $x$ in $M$ : this is call-by-name; or we first reduce $N$ to a result before doing the substitution: this is call-by-value.

In quantum computation, because of the non-duplicability of a quantum bit, the choice of reduction strategy might even render invalid a piece of code. For example, if $x$ links to a quantum bit, the code $(\lambda f x .(f x) x)($ meas $x)$ cannot be reduced right ahead to $\lambda f .(f(($ meas $x))(($ meas $x)$ as this duplicate the quantum bit. We first have to apply the measure, yielding a classical bit that can effectively be duplicated.

Generally speaking, in call-by-name a measurement of the form meas $M$ is carried over along $\beta$-reductions. There is no possible way to "force" the measurement to happen. However, it is possible to simulate a call-by-name reduction in call-by-value by encapsulating terms in lambda-abstractions. For example, to carry over the term meas $M$ using a call-by-value reduction strategy, one can write the term as $\lambda y$. meas $M$, with $y$ a fresh variable.

Because of this flexibility, in this quantum lambda-calculus we consider the call-by-value reduction strategy.

\section{[3.2.3] Reduction rules}

We then define a probabilistic call-by-value reduction procedure for the quantum lambda calculus. Note that, although the reduction itself is probabilistic, the choice of which redex to reduce at each step is deterministic. 


$$
\begin{gathered}
{[Q, L, \text { if } 0 \text { then } M \text { else } N] \rightarrow_{1}[Q, L, N]} \\
{[Q, L, \text { if } 1 \text { then } M \text { else } N] \rightarrow_{1}[Q, L, M]} \\
{\left[Q, L, U\left\langle x_{j_{1}}, \ldots, x_{j_{n}}\right\rangle\right] \rightarrow_{1}\left[Q^{\prime}, L,\left\langle x_{j_{1}}, \ldots, x_{j_{n}}\right\rangle\right]} \\
{\left[\alpha\left|Q_{0}\right\rangle+\beta\left|Q_{1}\right\rangle, L, \text { meas } x_{i}\right] \rightarrow_{|\alpha|^{2}}\left[\left|Q_{0}\right\rangle, L, 0\right]} \\
{\left[\alpha\left|Q_{0}\right\rangle+\beta\left|Q_{1}\right\rangle, L, \text { meas } x_{i}\right] \rightarrow_{|\beta|^{2}}\left[\left|Q_{1}\right\rangle, L, 1\right]} \\
{\left[Q,\left|x_{1} \ldots x_{n}\right\rangle, \text { new } 0\right] \rightarrow_{1}\left[Q \otimes|0\rangle,\left|x_{1} \ldots x_{n+1}\right\rangle, x_{n+1}\right]} \\
{\left[Q,\left|x_{1} \ldots x_{n}\right\rangle, \text { new } 1\right] \rightarrow_{1}\left[Q \otimes|1\rangle,\left|x_{1} \ldots x_{n+1}\right\rangle, x_{n+1}\right]}
\end{gathered}
$$

Table 1 Reductions rules of the quantum lambda calculus

A value is a term of the following form:

Value $V, W:=\quad x|\lambda x . M| 0|1| * \mid$ meas $\mid$ new $|U|\langle V, W\rangle$.

An abstract machine of the form $[Q, L, V]$ where $V$ is a value is called a quantum value-state.

The reduction rules are shown in Table 1 . We write $[Q, L, M] \rightarrow_{p}$ $\left[Q^{\prime}, L^{\prime}, M^{\prime}\right]$ for a single-step reduction of states which takes place with probability $p$. In the rule for reducing the term $U\left\langle x_{j_{1}}, \ldots, x_{j_{n}}\right\rangle, U$ is an $n$-ary built-in unitary gate, $j_{1}, \ldots, j_{n}$ are pairwise distinct, and $Q^{\prime}$ is the quantum state obtained from $Q$ by applying this gate to qubits $j_{1}, \ldots, j_{n}$. In the rule for measurement, $\left|Q_{0}\right\rangle$ and $\left|Q_{1}\right\rangle$ are normalized states of the form

$$
\left|Q_{0}\right\rangle=\sum_{j} \alpha_{j}\left|\phi_{j}^{0}\right\rangle \otimes|0\rangle \otimes\left|\psi_{j}^{0}\right\rangle, \quad\left|Q_{1}\right\rangle=\sum_{j} \beta_{j}\left|\phi_{j}^{1}\right\rangle \otimes|1\rangle \otimes\left|\psi_{j}^{1}\right\rangle,
$$

where $\phi_{j}^{0}$ and $\phi_{j}^{1}$ are $i$-qubit states (so that the measured qubit is the one pointed to by $\left.x_{i}\right)$. In the rule for new, $Q$ is an $n$-qubit state, so that $Q \otimes|i\rangle$ is an $(n+1)$ qubit state.

There are also $\xi$-rules for each term constructor, such as

$$
\frac{[Q, N] \rightarrow_{p}\left[Q^{\prime} L, N^{\prime}\right]}{[Q, L, M N] \rightarrow_{p}\left[Q^{\prime}, L, M N^{\prime}\right]} \quad \frac{[Q, M] \rightarrow_{p}\left[Q^{\prime}, L, M^{\prime}\right]}{[Q, L, M V] \rightarrow_{p}\left[Q^{\prime}, L, M^{\prime} V\right]}
$$

or

$$
\frac{[Q, L, P] \rightarrow_{p}\left[Q^{\prime}, L, P^{\prime}\right]}{[Q, L, \text { if } P \text { then } M \text { else } N] \rightarrow_{p}\left[Q^{\prime}, L, \text { if } P^{\prime} \text { then } M \text { else } N\right]}
$$

keeping in mind that we do not reduce under the branches of a test.

\section{[3.2.4] Type System}


The reduction system has run-time errors: the state $[Q, L, H(\lambda x . x)]$ and the state $[Q,|x y z\rangle, U\langle x, x\rangle]$ are both invalid. In the next section, we introduce a type system designed to rule out such erroneous states.

Types In the lambda calculus we just defined, we need to be able to account for higher-order, products, classical booleans and quantum booleans. Since we do not want to have specific term constructs for dealing with duplication and non-duplication, the information has to be encoded into the type system. We use a type system inspired from linear logic ${ }^{15)}$. By default, a term of type $A$ is assumed to be non-duplicable, and duplicable terms are given the type $! A$ instead.

Formally, we define the following type system for the quantum lambda calculus as follows:

$$
\text { qType } A, B \quad::=\quad \text { bit } \mid \text { qbit }|! A|(A \multimap B)|\top|(A \otimes B) .
$$

The constant types bit and qbit stand respectively for the classical and the quantum booleans; the type $! A$ is for duplicable elements of type $A$; the type $A \multimap B$ for functions from $A$ to $B$; the type $\top$ for result of commands; the type $A \otimes B$ for pairs of elements of types $A$ and $B$.

We call the operator "!" the exponential.

The typing rules will ensure that any value of type $! A$ is duplicable. However, there is no harm in using it only once; thus, such a value should also have type $A$. For that purpose, we use a subtyping relation to capture this notion: the subtyping is morally stating that $! A<: A$, and verifying the usual properties otherwise: $A \otimes B<: A^{\prime} \otimes B^{\prime}$ and $A^{\prime} \multimap B<: A \multimap B^{\prime}$ provided that $A<: A^{\prime}$ and $B<: B^{\prime}$.

Typing Rules A typing judgment is the given of a set $\Delta=\left\{x_{1}: A_{1}, \ldots, x_{n}\right.$ : $\left.A_{n}\right\}$ of typed variables, a term $M$ and a type $B$, written as $\Delta \triangleright M: B$. We call the set $\Delta$ the typing context, or simply the context.

Given two contexts $\Delta_{1}$ and $\Delta_{2}$, we write $\left(\Delta_{1}, \Delta_{2}\right)$ for the context consisting of the variables in $\Delta_{1}$ and in $\Delta_{2}$. When described in this form, it is assumed that $\left|\Delta_{1}\right| \cap\left|\Delta_{2}\right|=\emptyset$.

A typing derivation is called valid if it can be inferred from the rules of Table 2. We write $! \Delta$ for a context of the form $\left\{x_{1}: ! A_{1}, \ldots, x_{n}: ! A_{n}\right\}$. We use the notation $|\Delta|$ to represent the set of (untyped) variables $\left\{x_{1}, \ldots, x_{n}\right\}$ 
contained in $\Delta$. In the table, the symbol $c$ spans the set of term constants $\{$ meas, new $U, 0,1\}$. To each constant $c$ we associate a term $A_{c}$, as follows:

$A_{0}, A_{1}=b i t, A_{\text {new }}=b i t \multimap q b i t, A_{U}=q b i t^{\otimes n} \multimap q b i t^{\otimes n}, A_{\text {meas }}=q b i t \multimap ! b i t$.

The type $! A_{c}$ is understood as being the "most generic" one for $c$, as enforced by the typing rule $\left(a x_{2}\right)$. For example, we defined $A_{\text {new }}$ to be bit - qbit. Since new can take any type $B$ such that $! A_{c}<: B$, the term new can be typed with all the types in the poset:

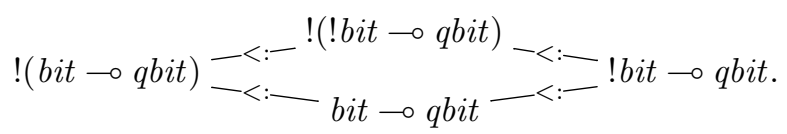

This implies, as expected, that no created quantum bit can have the type !qbit.

In general, the type system enforces the requirement that variables holding quantum data cannot be freely duplicated; thus $\lambda x .\langle x, x\rangle$ is not a valid term of type $q b i t \multimap q b i t \otimes q b i t$. On the other hand, we allow variables to be discarded freely.

Note also that due to rule $\left(\lambda_{2}\right)$ the term $\lambda x . M$ is duplicable only if all the free variables of $M$ (other that $x$ ) are duplicable. This follows the call-byvalue approach: since $\lambda x . M$ is a value, if it is duplicated it will be duplicated as a syntactic string of symbols, thus duplicating every single free variable of it (other than $x$ ).

Properties of the Type System An abstract machine [ $Q, L, M]$ can be said to be well-typed (or valid) of type $A$ as one could expect: $M$ needs to be of type $A$, and all the variables in $L$ are taken to be of type qbit.

The properties of the type system are then canonical: A well-typed program only reduces to well-typed programs of the same type, and if a program is well-typed, either it is a value or it reduces to some other program.

\section{[3.2.5] Example: quantum teleportation}

It turns out that some quantum algorithms can be efficiently understood as elements of higher-order types. We give in this section the implementation of the teleportation procedure as a higher-order term, despite the fact that the usual formalism is completely sequential ${ }^{30)}$.

The Teleportation Procedure Consider the teleportation algorithm as it is described in Section 4.3 (Part $\left.1^{30)}\right)$. We can embed each part of the procedure 


$$
\begin{gathered}
\frac{A<: B}{\Delta, x: A \triangleright x: B}\left(a x_{1}\right) \quad \frac{! A_{c}<: B}{\Delta \triangleright c: B}\left(a x_{2}\right) \\
\frac{\Gamma_{1}, ! \Delta \triangleright P: b i t \quad \Gamma_{2}, ! \Delta \triangleright M: A \quad \Gamma_{2}, ! \Delta \triangleright N: A}{\Gamma_{1}, \Gamma_{2}, ! \Delta \triangleright \text { if } P \text { then } M \text { else } N: A}(\text { if }) \\
\frac{\Gamma_{1}, ! \Delta \triangleright M: A \multimap B \quad \Gamma_{2}, ! \Delta \triangleright N: A}{\Gamma_{1}, \Gamma_{2}, ! \Delta \triangleright M N: B}(\text { app }) \\
\frac{\text { If } F V(M) \cap|\Gamma|=\emptyset:}{\Gamma: A, \Delta \triangleright M: B}\left(\lambda_{1}\right) \quad \frac{\Gamma, ! \Delta, x: A \triangleright M: B}{\Gamma, ! \Delta \triangleright \lambda x \cdot M: !^{n+1}(A \multimap B)}\left(\lambda_{2}\right) \\
\frac{! \Delta, \Gamma_{1} \triangleright M_{1}: !^{n} A_{1} \quad ! \Delta, \Gamma_{2} \triangleright M_{2}: !^{n} A_{2}}{! \Delta, \Gamma_{1}, \Gamma_{2} \triangleright\left\langle M_{1}, M_{2}\right\rangle: !^{n}\left(A_{1} \otimes A_{2}\right)}(\otimes . I) \quad \overline{\Delta \triangleright *: ! \top}(\top . I) \\
\frac{! \Delta, \Gamma_{1} \triangleright M: !^{n}\left(A_{1} \otimes A_{2}\right) \quad ! \Delta, \Gamma_{2}, x_{1}: !^{n} A_{1}, x_{2}: !^{n} A_{2} \triangleright N: A}{! \Delta, \Gamma_{1}, \Gamma_{2} \triangleright \text { let }\left\langle x_{1}, x_{2}\right\rangle=M^{\prime} \text { in } N: A}(\otimes . E)
\end{gathered}
$$

Table 2 Typing rules

as a function. There is a function EPR $: !(\top \multimap(q b i t \otimes q b i t))$ that creates an entangled state, as in Step (1):

$$
\mathbf{E P R}=\lambda x . C N O T\langle H(\text { new } 0), \text { new } 0\rangle .
$$

There is a function BellMeasure $: !(q b i t \multimap(q b i t \multimap b i t \otimes b i t))$ that takes two qubits, rotates and measures them, as in Step (2):

BellMeasure $=\lambda q_{2} . \lambda q_{1} .\left(\right.$ let $\langle x, y\rangle=\operatorname{CNOT}\left\langle q_{1}, q_{2}\right\rangle$ in $\langle$ meas $(H x)$, meas $y\rangle$

We also can define a function $\mathbf{U}: !(q b i t \multimap(b i t \otimes b i t \multimap q b i t))$ that takes a qubit $q$ and two bits $x, y$ and returns $U_{x y} q$, as in Step (3):

$$
\begin{array}{r}
\mathbf{U}=\lambda q \cdot \lambda\langle x, y\rangle . \text { if } x \text { then (if } y \text { then } U_{11} q \text { else } U_{10} q \text { ) } \\
\text { else (if } y \text { then } U_{01} q \text { else } U_{00} q \text { ). }
\end{array}
$$

The teleportation procedure can be seen as the creation of two non-duplicable functions $f$ and $g$

$$
f: q b i t \multimap b i t \otimes b i t, \quad g: b i t \otimes b i t \multimap q b i t,
$$


such that $(g \circ f)(x)=x$ for an arbitrary qubit $x$. We can construct such a pair of functions by the following code:

$$
\text { let } \begin{aligned}
\langle x, y\rangle & =\mathbf{E P R} * \text { in } \\
\text { let } f & =\mathbf{B e l l M e a s u r e ~} x \text { in } \\
\text { let } g & =\mathbf{U} y \\
\text { in }\langle f, g\rangle . &
\end{aligned}
$$

Note that, since $f$ and $g$ depend on the state of the qubits $x$ and $y$, respectively, these functions cannot be duplicated, which is reflected in the fact that the types of $f$ and $g$ do not contain a top-level "!".

\section{[3.2.6] Shortcomings of the language}

This quantum lambda-calculus provides a sound and unified framework for manipulating classical and quantum data. It also makes it possible to potentially define oracles as higher-order functions. The language successfully addresses the issue of the distinction between duplicable and non-duplicable data, even at higher-order type, and do so in a general manner. Finally, it can easily be extended with a richer type system (such as lists) and recursion ${ }^{29)}$.

However, it has several shortcomings that cannot be overcome with a simple extension: first, if it is possible to write and manipulate oracles as firstclass objects, there is no way to automatically make a unitary map out of a classical description: the only unitaries around are the elementary ones. It also lacks a way of manipulating quantum circuits. For example, one cannot reverse a computation. It is not possible to process a quantum circuit to remove redundancies, or describe and apply optimization. It is neither possible to apply an error correcting code to a given function within the language.

\subsection{A toy language for circuit construction}

The shortcomings described in Section 3.2.6 are mainly due to the fact that the semantics of the quantum lambda-calculus does not separate circuit construction from circuit evaluation. The quantum IO monad of Green and Altenkirch ${ }^{17)}$ proposes an alternative approach for dealing with quantum computation, and the technique addresses this particular problem.

The language follows an intuition different from the one developed in the quantum lambda-calculus.

1. First, the language is a language embedded in a host language, Haskell. 
The choice of the host language has its importance: Haskell is a higherorder, functional language with a rich type system (much richer than the ML type-system, for example). This makes it a good candidate for hosting an embedded language while giving it a well-defined operational semantics.

2. The language separates the construction of a quantum computation from its execution. While being built, the computation is stored in a datastructure that one could then theoretically manipulate. As such, the language only proposes commands to run various simulations, but the implementation is modular enough to imagine being able to add a "run" command to hook on a real quantum computer, or add some commands to perform code rewriting.

3. The other noteworthy feature of the quantum IO monad is the use of Haskell's rich type system to define a notion of generic quantum data. With a single term construct, one can define the measure of a tuples of quantum bits, or a list of quantum bits, or a tuples of list of quantum bits... This gives a concrete definition of the otherwise vaporous notion of quantum data.

In the following, we give a brief tutorial to the quantum IO monad, illustrating the features that are worth having in a quantum programming language. Although the language being embedded in Haskell, we'll try our best to make the exposition self-contained. Haskell code is written in typewriter font.

\section{[3.3.1] An embedded language in Haskell}

Standing on top of Haskell, the language comes with three main specific type constructors: a type of quantum bits Qbit, a type of unitary maps $\mathrm{U}$, and a type constructor for quantum computation QIO. A function of type A $\rightarrow$ QIO B is understood as a function from an object of type A, returning a quantum computation whose result would be of type B. The type constructor QIO records the fact that some non-purely functional operations are taking place along the evaluation of the function.

Elementary quantum computation. The basic blocks of quantum computation are represented as Haskell functions.

- measQbit :: Qbit -> QIO Bool. For measurements: a function taking a qubit as input and outputting a classical boolean. 
- mkQbit : : Bool -> QIO Qbit. For creating quantum bits: a function taking a classical boolean as input and producing a quantum bit.

- applyU :: U $\rightarrow$ QIO (). For unitaries: it is a command taking a unitary matrix as input, and producing... a quantum computation. A command is a function of output type void, represented by the empty tuple () in Haskell.

Building unitaries. How are we to build unitaries of type U? The following operations are at available.

- swap : : Qbit $\rightarrow$ Qbit $\rightarrow$ U. For swapping the quantum bits referenced by the two inputs.

- cond : : Qbit $\rightarrow$ (Bool $\rightarrow$ U) $\rightarrow$ U. For dynamically constructing of controlled operation. This operation is nothing else than an if-then-else construction for quantum bit, as it was already proposed in $\mathrm{QML}^{2)}$.

- $\operatorname{rot}::$ Qbit $\rightarrow((\mathrm{Bool}, \mathrm{Bool}) \rightarrow \mathbb{C}) \rightarrow \mathrm{U}$. The first input is a reference to a quantum bit, and the second input is the description of a $2 \times 2$ matrix. This operation constructs the circuit applying the matrix to the qubit.

- ulet : : Bool $\rightarrow$ (Qbit $\rightarrow$ U) $\rightarrow$ U. Allocation of auxiliary ancillas. First the ancilla is created with the value of the first input, then the second argument is applied to this ancilla. Finally, the ancilla is erased (i.e. measured).

- urev :: U $\rightarrow$ U. Reverse the circuit description given in input.

Finally, the type $\mathrm{U}$ is enriched with the notion of monoid (thanks to Haskell's typeclass Monoid). Practically, this means that two other useful constructions are available: mempty : : $\mathrm{U}$, representing the empty circuit and mappend : : $\mathrm{U} \rightarrow \mathrm{U} \rightarrow \mathrm{U}$, representing sequencial composition of circuits.

Example of unitary constructions. The Hadamard gate, using an auxilliary function:

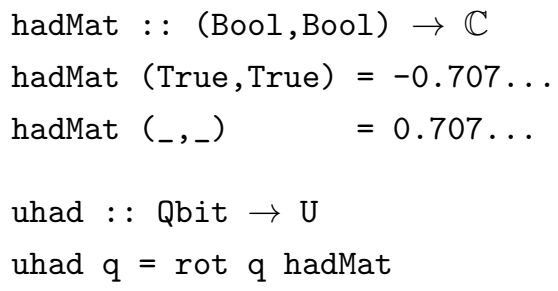


One can define a generic control operator as a one-sided if-construction:

ifQ : : Qbit $\rightarrow \mathrm{U} \rightarrow \mathrm{U}$

if $\mathrm{q} u=$ cond $\mathrm{q}(\lambda \mathrm{x} \rightarrow$ if $\mathrm{x}$ then $\mathrm{u}$ else mempty)

Using lists of quantum bits (in Haskell, [A] is the notation for a list of elements of type A), one can use the programmable feature of the circuit construction and define a multi-control operation with recursion.

ifQx : : [Qbit] $\rightarrow \mathrm{U} \rightarrow \mathrm{U}$

ifQx [] $u=u$

ifQx (h:t) $u=\operatorname{ifQ} h($ ifQx $t u)$

Arguably, this program is implementing a multi-controlled gate in a "natural" way.

General quantum computation. Haskell proposes an imperative style way for composing operations of type QIO (thanks to the monadic interface). Consider the two functions $\mathrm{f}: \mathrm{:} \quad \mathrm{A} \rightarrow$ QIO $\mathrm{B}$ and $\mathrm{g}:: \mathrm{B} \rightarrow$ QIO C. The composition of $f$ and $g$ is done using the syntax

$\mathrm{h}: \mathrm{A} \rightarrow \mathrm{QIO} \mathrm{C}$

$\mathrm{h} x=$ do $\mathrm{y}<-\mathrm{f} \mathrm{x} ; \mathrm{z}<-\mathrm{g} \mathrm{y} ; \operatorname{return} \mathrm{z}$

The syntax is self explanatory: store the result of $\mathrm{f} \mathrm{x}$ in $\mathrm{y}$, feed it to $\mathrm{g}$, get $z$, finally return $z$. One can of course compose many operations in one go, or apply commands of type QIO (), extending the syntax in the obvious way. For example, the coin-toss can be defined as follows.

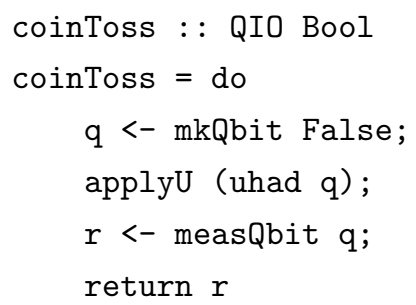

Quantum teleportation. As a complete example, we show the implementation of the quantum teleportation algorithm ${ }^{17)}$. Compared to the quantum lambda-calculus, the main differences is that every function returns a computation QIO. We assume that unot is the gate $N$ and that uZ is the gate $Z$. 


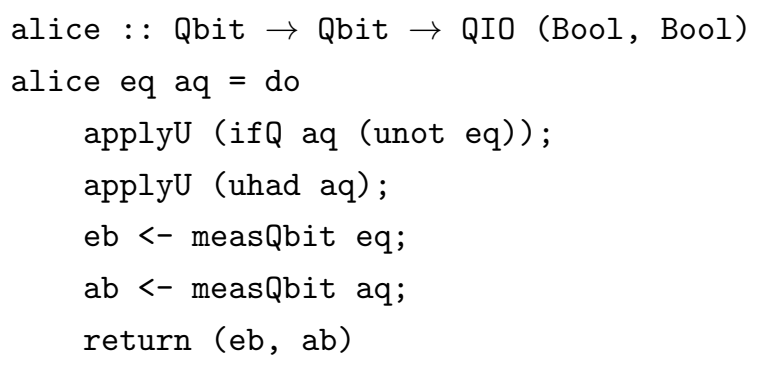

Provided that bobsU : : (Bool, Bool) $\rightarrow$ Qbit $\rightarrow$ U implements the map $(x, y) \mapsto$ $U_{x y}$, we define the action of Bob as

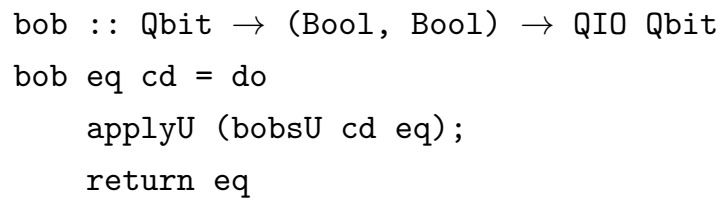

We write the teleportation algorithm as in Section 3.2.5. Here, the type of the term is a bit more complex due to the construct QIO.

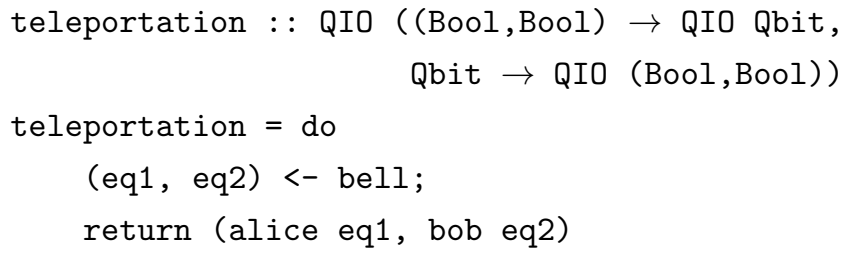

It is of course possible to compose the two functions, and any reasonnable operational semantics would produce an identity map.

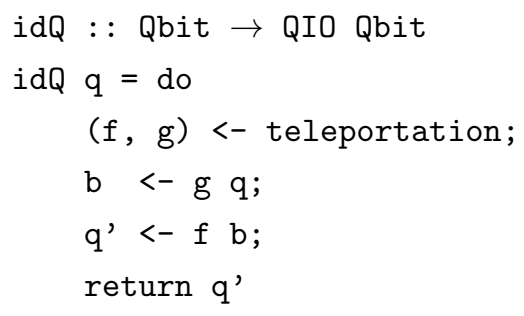

\section{[3.3.2] Typeclasses and quantum data}

A particularly useful feature in the quantum IO proposal is the notion of generic quantum data. Thanks to Haskell's typeclasses, one can overload the type of a function and still stay in a strongly typed paradigm. A typeclass is a 
property of a type to which is attached a set of functions. In the case of quantum data, the quantum IO monad proposes

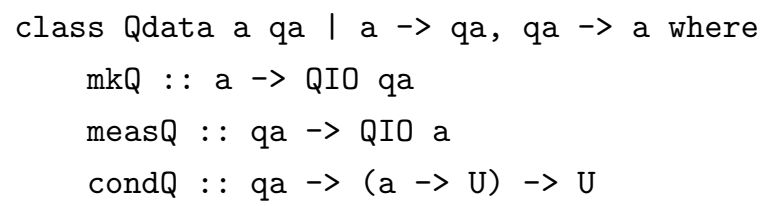

That is, the typeclass Qdata place in relation two types a and qa, and define for each pair ( $a, q a)$ three functions mkQ, measQ and condQ. The names of these functions are deliberately close to the maps mkQbit, measQ and cond, since the first pair (a,qa) is (Bool, Qbit):

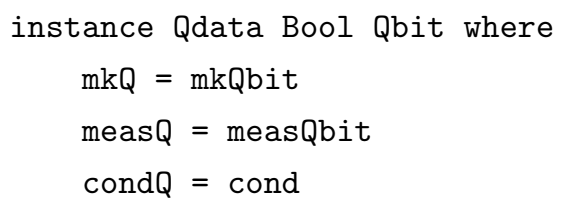

Provided that (a,qa) and ( $b, q b)$ are already member of Qdata, we can also make ( $(a, b),(q a, q b))$ member of Qdata:

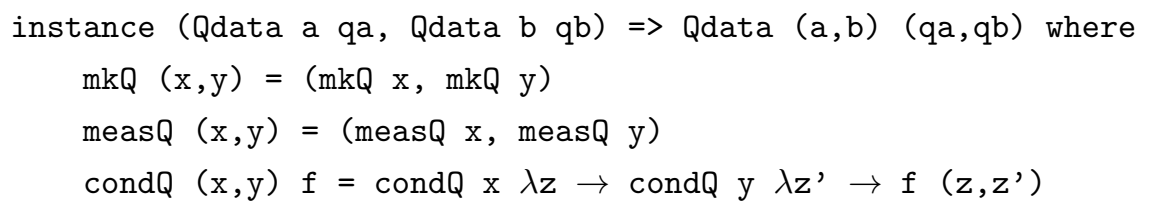

Similarly, it is possible to add tuples, and lists in the scheme. But one can make more "interesting" correspondences; for example, if we define the type data QInt $=$ QInt [Qbit]

one can make (Int, QInt) into an instance of Qdata (by fixing the number of digits a QInt is allowed to have, and by choosing whether we are little or big endian), so that mkQ 4 for example builds the state $|\ldots 0100\rangle$

\section{[3.3.3] Discussion}

The quantum IO monad offers a versatile way of manipulating pieces of quantum algorithms: quantum circuits on one hand, and quantum computation in general on another hand. It also features a systematic procedure to extend quantum datatypes. These ideas are to keep in mind while designing a scalable quantum programming language. 
However, several drawbacks hamper the language, and does not make it the perfect fit we were looking for.

1. The type of unitaries $U$ is untyped. As such, a unitary is a blackbox that does not contain any information on the structure of the input and the output. Unitaries cannot easily be used with Qdata.

2. The typeclass Qdata leaves the programmer frustrated: the correspondence classical-quantum is only drawn for elementary functions: how about producing a map QInt $\rightarrow$ QInt out of a map Int $\rightarrow$ Int?

3. Despite its power, Haskell's type system is not capable of forbidding duplication of quantum bits. For example, the following buggy code is nonetheless valid:

copy : : Qbit $\rightarrow$ QIO (Qbit, Qbit)

copy $q=$ do $\operatorname{return}(q, q)$

This is certainly problematic in the light of our requirements for a quantum programming language.

\subsection{Monads and quantum computation}

Albeit kept under the shed, in the two languages we reviewed a structuring notion kept showing up: the notion of monad. Monads are semantic tools to capture and reason about side-effects.

Side-effects appear in a language when the notion of value, or result is distinct from the notion of computation. Exemples of side-effects include.

- Probabilistic behavior. If there is a way to toss a coin in a language, the coin-toss is operationally distinct from its result.

- Input-output. If the language possesses a library for accessing a terminal, or a tape, or a network, or a file-system..., sure enough a computation is not similar to a result.

- State. If references, or pointers are available in the language, this can often be represented by a monad.

These three side-effects appear in the situations we explored:

- QIO is can be regarded as input-output,

- $\mathrm{U}$ is a state monad,

- the measurement induces a probabilistic side-effect. 
In the quantum lambda-calculus, the operational semantics makes use of an explicit state monad to keep track of the quantum bits already allocated. However, thanks to the type system enforcing linearity on quantum bits, a computation involving unitaries (such as $H(N x)$ ) is the same thing as the result of the computation (since one can only use it once): in the quantum lambda-calculus, the quantum bits is not the part breaking the pure functionality of the language.

The important aspects these two languages teach us is that

- linear type systems can efficiently deal with the non-duplicability of quantum information;

- monads are probably the good paradigm to manipulate quantum circuits and quantum computation as a piece of data.

- generic quantum data definable by the programmer makes sense, but should be used in parallel with automatic classical-to-quantum conversion to take full advantage of their genericity.

\subsection{Semantics of quantum computation}

A second research area is the development of semantics for quantum computation. The goal is two-fold. First, understanding the structure of quantum information ${ }^{13)}$. Then, developping tools for specification and verification of quantum algorithms. In the event of quantum computers, these tools might reveal as useful as they were for classical computation ${ }^{32}$.

\section{[3.5.1] Completely positive maps}

A well-known fact ${ }^{22}$ is that vectors in Hilbert spaces do not form a satisfying framework to describe quantum computation when it involves measurement. Consider the two computations

$$
\begin{gathered}
\text { new }(\text { meas }(H(\text { new } 0))), \\
H(\text { new }(\text { meas }(H(\text { new } 0))) .
\end{gathered}
$$

The former outputs the equal probability distribution consisting of the states $|0\rangle$ and $|1\rangle$ whereas the latter outputs the equal probability distribution consisting of the states $\frac{1}{\sqrt{2}}(|0\rangle+|1\rangle)$ and $\frac{1}{\sqrt{2}}(|0\rangle-|1\rangle)$. One can argue that these are distinct. However, this is not the case: there are observationally equivalent, i.e. one cannot find a computation that can distinguish between them. For example, if you simply measure them with respect to the standard basis, they both answer 0 and 1 with equal probability. Intuitively, the idea is that a quantum computation 
on either state is morally "just" a general measurement, and one can generalize this idea to build a denotational semantics for quantum computation.

A representation that is invariant under this observational equivalence is the notion of positive matrix. There are several equivalent definitions for positive matrices. The one of interest in our situation is this one:

\section{Definition 3.1}

A positive matrix is a square-matrix of the form $\sum_{i=1}^{n} \rho_{i} u_{i} u_{i}^{*}$ where the $\rho_{i}$ 's are non-negative reals, the $u_{i}$ 's are normalized vectors of same dimension. The notation $u_{i}^{*}$ represents the conjugate transpose of $u_{i}$. That is, if $u_{i}$ is the 2dimensional vector $\left(\begin{array}{c}\alpha \\ \beta\end{array}\right), u_{i}^{*}$ is the row-vector $(\bar{\alpha} \bar{\beta})$. The element $u_{i} u_{i}^{*}$ is then the $2 \times 2$ matrix $\left(\begin{array}{cc}\alpha \bar{\alpha} & \alpha \bar{\beta} \\ \beta \bar{\alpha} & \beta \bar{\beta}\end{array}\right)$.

One can therefore represent a probabilistic superposition of states by taking the $\rho_{i}$ 's as the probability for a particular state $u_{i}$ to be the result of the computation. Then in the above example, the output of Program (2) is

$$
\frac{1}{2}\left(\begin{array}{l}
1 \\
0
\end{array}\right)\left(\begin{array}{ll}
1 & 0
\end{array}\right)+\frac{1}{2}\left(\begin{array}{l}
0 \\
1
\end{array}\right)\left(\begin{array}{ll}
0 & 1
\end{array}\right)=\left(\begin{array}{cc}
\frac{1}{2} & 0 \\
0 & \frac{1}{2}
\end{array}\right),
$$

whereas the output of Program (3) is

$$
\frac{1}{2}\left(\begin{array}{c}
\frac{1}{\sqrt{2}} \\
\frac{1}{\sqrt{2}}
\end{array}\right)\left(\frac{1}{\sqrt{2}} \frac{1}{\sqrt{2}}\right)+\frac{1}{2}\left(\begin{array}{c}
\frac{1}{\sqrt{2}} \\
\frac{-1}{\sqrt{2}}
\end{array}\right)\left(\frac{1}{\sqrt{2}} \frac{-1}{\sqrt{2}}\right)=\frac{1}{2}\left(\begin{array}{cc}
\frac{1}{2} & \frac{1}{2} \\
\frac{1}{2} & \frac{1}{2}
\end{array}\right)+\frac{1}{2}\left(\begin{array}{cc}
\frac{1}{2} & \frac{-1}{2} \\
\frac{-1}{2} & \frac{1}{2}
\end{array}\right)=\left(\begin{array}{cc}
\frac{1}{2} & 0 \\
0 & \frac{1}{2}
\end{array}\right) .
$$

Their representation in term of positive matrices is the same: this is consistent with the fact that the two programs are indistinguishable.

If the result of a computation is a positive matrix, a computation is a completely positive map. That is:

- sending positive matrices to positive matrices;

- linear, in the sense of linear algebra: if the map is $f, f(x+y)=f(x)+f(y)$ and $f(\rho \cdot x)=\rho \cdot f(x)$.

If we deal with first-order maps (i.e. with inputs and outputs being only classical and quantum bits), one can also add

- trace-preserving,

in which case the map is called a superoperator.

Denotational semantics. A denotational semantics for a programming language is a mathematical representation of its programs. A program is regarded 
as a process inputting some data and outputting some other data: its semantics is a function whose domain is the datatype of input and whose codomain is the datatype of output.

The semantics of a programming language can have three properties: adequacy, full-abstraction and full completeness. If $\llbracket P \rrbracket$ represents the denotation of the program $P$, we can define these notions as follows ${ }^{32}$.

- Let $\Omega$ be the diverging program. The semantics is adequate if whenever $\llbracket P \rrbracket=\llbracket \Omega \rrbracket$ where $\Omega$ is the looping program, then $P$ is also nonterminating.

- The semantics is fully-abstract if for all programs $P$ and $Q, \llbracket P \rrbracket=\llbracket Q \rrbracket$ if and only if $P$ and $Q$ are observationally equivalent. A potential use for a fully abstract semantics is to validate code optimization, for example in the context of the construction of a compiler.

- Finally, the semantics is fully complete if moreover, every element $v$ in the image of a type $A$ corresponds to a program $P$. With such a semantics, one has a decision tool that can determine using mathematical methods whether a given set of specifications can be realized by a program.

Quantum lambda-calculus. Positive matrices and completely positive maps form a denotational for the quantum lambda-calculus in the following sense.

- Completely positive maps form a fully-abstract semantics for the strictly linear fragment of the quantum lambda-calculus ${ }^{27)}$ : the model only represents programs whose types do not contain any instance of "!". In particular, if $f$ and $g$ are the two functions built in Section 3.2.5, then $g \circ f: q b i t \rightarrow q b i t$ has the same denotation as the identity function $\lambda x . x$.

- Superoperators form a complete semantics for the first-order fragment of the stricly linear lambda-calculus ${ }^{26}$. This means that any superoperator can be regarded as a program unique up to observational equivalence, and vice-versa.

\section{[3.5.2] Linear logic}

Completely positive maps does not capture the whole quantum lambdacalculus: duplication is not handled. One can wonder what is the structure of the type operator "!", and what are the properties it satisfies.

Programs and Proofs. Through the Curry-Howard correspondence ${ }^{19)}$, the type of a program can be interpreted as a proposition in a particular logic. The 
program corresponds to the proof of the proposition, and the cut-elimination of the proof corresponds to the evaluation of the program. A rich enough logic admits a notion of conjunction $\wedge$ and a notion of implication $\Rightarrow$. In the type system, these correspond to pairing and function types. In the realm of the semantics, the connectives $(\wedge, \Rightarrow)$ forms an adjunction, a powerful categorical notion.

The notions of semantics, typed languages and logical systems interact in complex ways, each one giving insight on the structure of the other ones.

A modality for non-linearity. Linear logic is a resource-sensitive logic introduced by Girard ${ }^{15)}$. This logic acknowledges the fact that the structural rules are usually hidden in regular, classical logic: for example, the introduction rule for the conjunction can be decomposed in two distinct ways, giving rise to two distinct conjunctions: a multiplicative $\otimes$ and an additive $\&$ :

$$
\frac{\Delta \triangleright A \quad \Gamma \triangleright B}{\Delta, \Gamma \triangleright A \otimes B} \quad \frac{\Delta \triangleright A \quad \Delta \triangleright B}{\Delta \triangleright A \& B}
$$

In order to go from one to the other, one needs to apply two structural rules: weakening and contraction:

$$
\frac{A \triangleright C}{A, B \triangleright C} \quad \frac{A, A \triangleright B}{A \triangleright B}
$$

In linear logic, structural rules can only be applied on formulas of the form ! $A$. In general there are four structural rules: weakening and contraction, but also dereliction and promotion, respectively:

$$
\frac{A \triangleright C}{A, ! B \triangleright C}, \quad \frac{! A, ! A \triangleright B}{! A \triangleright B}, \quad \frac{A \triangleright B}{! A \triangleright B}, \quad \frac{! A \triangleright B}{! A \triangleright ! B} .
$$

The modality "!" can be regarded as the duplicability flag in the type system of the quantum lambda-calculus: weakening and promotion restricts which data can be erased and duplicated, whereas derelication and promotion relate duplicable data with non-duplicable data.

A valid typing judgement in the quantum lambda-calculus can therefore be seen as a proof in a linear-logic-like logical system. It does not match precisely linear logic because the type operator "!" is weaker: in particular, !! $A$ is a type equivalent to $! A$, which is not the case in linear logic. 
Relation with models. If the two logical systems do not match precisely, they match enough to be able to adapt models of linear logic to the subset of quantum computation described by the quantum lambda-calculus. Specically, many models of linear logic are based on functional analysis and theory of operator spaces, and use the algebraic linearity to reflect the logical linearity ${ }^{10,5,12,11)}$. The spaces used for the models are rich enough to be able to generate the modalities "!" with the correct behavior. It has been shown that in some context it is possible to use the constructions to make models of quantum computation ${ }^{16,18)}$.

\section{[3.5.3] Graphs and MBQC}

An successful alternative approach to verify properties of quantum programs is the use of rewriting rules in graphical representation of symmetric monoidal and compact closed categories. Although this is particularly relevant in the paradigm of measurement-based quantum computation, it is a tool to keep an eye on when concerned with optimization of quantum circuits.

Categorical representation. Entanglement structures such as the one designed at the beginning of a computation in the MBQC model (Section 2.1) can be regarded simply as a graph: called graph state, they possess many properties that can be abstracted away and described in categorical models ${ }^{9)}$.

The canonical categorical models that can be used to described quantum computation are the compact closed structures ${ }^{1)}$ : for our purpose, their most interesting aspect is that they feature a graphical language, and equations in compact closed structures can be set in correspondence with rewrite rules in the graphical language.

Playing with additional structures on compact closed categories allows one to describe with sharp precision many constructions in quantum computation, in particular with what can be done with graph states. A partially automated tool, Quantomatic ${ }^{8}$, have been developped for helping the reasonning in this theoretical framework. It is readily available to help analyzing circuit optimizations.

\section{$\S 4$ Conclusion}

Quantum computation is yet at an early stage: there does not yet exist a technology to build scalable quantum computers, and the development is hindered by the unstability of quantum information. 
However, if the technology is not yet up to scale, the theory behind quantum computation is well-understood. This is calling for a theoretical computer science to be developed, using all the insight computer science have been developing for classical computation. Semantical tools are getting shaped: it is time to develop quantum programming languages and analysis techniques to get ready for the birth of scalable quantum computers.

This is an exciting field with many open questions and extremely diverse problematics. We hope this review raised some interest among the readers: this computational paradigm is still vastly unexplored, and it is calling for expertize from all domains of computer science.

\section{References}

1) Abramsky, S. and Coecke, B., "A categorical semantics of quantum protocols," in Proc. of LICS'04, pp. 415-425, 2004.

2) Altenkirch, T. and Grattage, J., "A functional quantum programming language," in Proc. of LICS'05, pp. 249-258, 2005.

3) Barendregt, H. P., The Lambda-Calculus, its Syntax and Semantics, North Holland, 1984.

4) Bettelli, S., Calarco, T. and Serafini, L., "Toward an architecture for quantum programming," The European Physical Journal D, 25, 2, pp. 181-200, 2003.

5) Blute, R. F., Cockett, J. R. B. and Seely, R. A. G., "Differential categories," Math. Struct. in Comp. Sc., 16, 6, pp. 1049-1083, 2006.

6) Broadbent, A. and Kashefi, E., "Parallelizing quantum circuits," Th. Comp. Sc., 410, 26, pp. 2489-2510, 2009.

7) Danos, V., Kashefi, E., Panangaden, P. and Perdrix, S., "Extended measurement calculus," Ch. $7^{13)}$, pp. 235-310.

8) Dixon, L. and Duncan, R., "Graphical reasoning in compact closed categories for quantum computation," Annals of Math. and Art. Int., 56, pp. 23-42, 2009.

9) Duncan, R. and Perdrix, S., "Rewriting measurement-based quantum computations with generalised flow," in Proc. of ICALP'10, pp. 285-296, 2010.

10) Ehrhard, T., "On Köthe sequence spaces and linear logic," Math. Struct. in Comp. Sc., 12, 5, pp. 579-623, 2002.

11) Ehrhard, T., "Finiteness spaces," Math. Struct. in Comp. Sc., 15, 4, pp. 615-646, 2005.

12) Ehrhard, T. and Regnier, L., "The differential lambda-calculus," Th. Comp. Sc., 309, 1-2, pp. 1-41, 2003.

13) Gay, S. J. and Mackie, I., editors, Semantic Techniques in Quantum Computation, Cambridge University Press, 2009.

14) Gay, S. J., "Quantum programming languages: Survey and bibliography," Math. Struct. in Comp. Sc., 16, 4, pp. 581-600, 2006. 
15) Girard, J. Y., "Linear logic," Th. Comp. Sc., 50, 1, pp. 1-101, 1987.

16) Girard, J. Y., "Between logic and quantic: A tract," in Linear logic in computer science, Cambridge University Press, 2004.

17) Green, A. and Altenkirch, T., "The quantum IO monad," Ch. $5^{13)}$, pp. 173-205.

18) Hasuo, I. and Hoshino, N., "Semantics of higher-order quantum computation via geometry of interaction," in Proc. of LICS'11, pp. 237-246, 2011.

19) Howard, W., "The formulae-as-types notion of construction," in To H.B. Curry: essays on combinatory logic, lambda calculus, and formalism, Academic Press, pp. 479-490, 1980.

20) Kaye, P., Laflamme, R, and Mosca M., An Introduction to Quantum Computing, Oxford University Press, 2007.

21) Knill, E. H., "Conventions for quantum pseudocode," Tech. Rep. LAUR-962724, Los Alamos National Laboratory, 1996.

22) Nielsen, M. A. and Chuang, I. L., Quantum Computation and Quantum Information, Cambridge University Press, 2002.

23) Ömer, B., Quantum programming in QCL, Master's thesis, Institute of Information Systems, Technical University of Vienna, 2000.

24) Raussendorf, R. and Briegel, H. J., "A one-way quantum computer," Phys. Rev. Lett., 86, 22, pp. 5188-5191, 2001.

25) Sanders, J. W. and Zuliani, P., "Quantum programming," in Proc. of MPC'00, pp. 80-99, 2000.

26) Selinger, P., "Towards a quantum programming language," Math. Struct. in Comp. Sc., 14, 4, pp. 527-586, 2004.

27) Selinger, P. and Valiron, B., "On a fully abstract model for a quantum linear functional language," pp. 123-137.

28) Selinger, P. and Valiron B., "A lambda calculus for quantum computation with classical control," Math. Struct. in Comp. Sc., 16, 3, pp. 527-552, 2006.

29) Selinger P. and Valiron, B., "Quantum lambda-calculus," Ch $4^{13)}$, pp. 135-172.

30) Valiron, B., "Quantum computation: a tutorial," New Generation Computing, 2012. To appear.

31) van Tonder, A., "A lambda calculus for quantum computation," SIAM Journal of Computing, 33, 5, pp. 1109-1135, 2004.

32) Winskel, G., The Formal Semantics of Programming Languages, MIT Press, 1993. 